\title{
Aktuelle Trends in der Telemedizin
}

Die Telemedizin hat u. a. zum Ziel, die gesundheitliche Versorgung von Patienten über räumliche Distanzen hinweg zu ermöglichen. E. Ray Dorsey und Eric J. Topol haben nun am Beispiel der USA aktuelle und zukünftige Trends in der Telemedizin analysiert und Barrieren hinsichtlich deren Implementierung identifiziert.

Dorsey ER, Topol E]. State of Telehealth. N Engl ] Med 2016; 375: 154-61

Folgende Trends sind bei der Telemedizin aktuell von Bedeutung:

- Neben dem ursprünglichen Ziel der Telemedizin, den Zugang zu medizinischer Versorgung zu verbessern, soll diese zukünftig eine komfortablere Patientenversorgung ermöglichen und eventuell zu einer Kostenreduktion führen.

- Erweiterung des Anwendungsbereichs der Telemedizin, um diese neben der Behandlung von akuten Erkrankungen auch bei episodischen und chronischen Krankheiten verfügbar zu machen.

- Verlagerung der Telemedizin vom Krankenhaus und assoziierten Kliniken in das Zuhause der Patienten.

Aktuell stellt die Kostenrückerstattung im Zusammenhang mit der Telemedizin ein Hemmnis für die flächendeckende Anwendung der Technik dar. Der Versicherungsschutz ist derzeit lückenhaft, wobei allerdings ein positiver Trend besteht. So existieren nun beispielsweise in 29 Staaten (in doppelt so vielen wie vor 3 Jahren) Gesetze, die private Versicherer dazu verpflichten, Telemedizin-Dienstleistungen im gleichen Ausmaß abzudecken wie Dienstleistungen in der individuellen Versorgung.

\section{Weitere Hindernisse identifiziert \\ $\nabla$}

Es existieren aber noch weitere Barrieren für die Telemedizin. Dazu gehören beispielsweise klinische Barrieren, wie die Qualität der Arzt-Patienten-Beziehung, der Untersuchungen sowie der Pflege. Studien müssen deshalb zeigen, dass eine medizinische Betreuung über räumliche Distanzen hinweg tatsächlich die medizinischen Outcomes verbessert. Auch juristische Hindernisse wirken der Telemedizin entgegen. Stichwort Approbation: Der Arzt muss in der Regel in dem Bundesstaat (Land), in dem sich der zu behandelnde Patient befindet, zugelassen sein. Länderübergreifende Behandlungen sind in den USA nicht ohne Weiteres möglich. Weiterhin von juristischer Bedeutung sind beispielsweise Fragen zur Qualifikationsprüfung und Haftung. Das bedeutsamste Hemmnis für die Ausbreitung der Telemedizin ist aber möglicherweise sozialer $\mathrm{Na}$ tur. Für ältere Personen, solche in ländlichen Gegenden, mit geringer Bildung bzw. geringem Einkommen und chronischen Erkrankungen besteht eine höhere Wahrscheinlichkeit, dass diese keinen Internetzugang besitzen. So untergräbt insbesondere die digitale Kluft zwischen Bevölkerungsgruppen das Hauptziel der Telemedizin, nämlich vor allem den bedürftigsten Menschen einen Zugang zur Gesundheitsversorgung zu ermöglichen.

\section{Fazit}

Trotz ungelöster Probleme im Bereich der Finanzierung sowie substanzieller Barrieren ist im Fall der Telemedizin ein stetiges Wachstum zu verzeichnen. Nach Meinung der Autoren wird eine zunehmende Zahl von Kostenrückerstattungsmodellen die Ausbreitung der Telemedizin weiter fördern. Soziale Faktoren, die den Zugang der Menschen zum Internet beeinflussen, seien aber in diesem $\mathrm{Zu}-$ sammenhang möglicherweise von größerer Bedeutung, so die Autoren weiter.

Dr. Frank Lichert, Weilburg 